\title{
SNP Market View: Opportunities, Technologies, and Products
}

\author{
Matthew Wygant \\ Matthew Wygant Biotech Writing, Los Gatos, CA, USA
}

BioTechniques 32: S78-S86 (June 2002)

\section{INTRODUCTION}

Single nucleotide polymorphisms (commonly referred to as SNPs) are individual sites of nucleotide base variation in DNA. The human genome contains over ten million SNPs that occur across populations at an appreciable frequency $(3,8,12)$. Often, different SNPs are one of the major components of the genome responsible for the variations we see between individuals. However, many different SNPs also contribute to the genetic component of variations between individuals that we don't see, such as susceptibility to certain diseases and responsiveness to drug therapies (see M. de Arruda, this Supplement).

Pharmacogenomics will allow us to use SNPs to provide better medicines to patients. Being able to predict responsiveness to drug therapy will help us to avoid the astonishing number of hospitalizations and deaths attributable to adverse drug reactions; as an example, a 1998 meta-analysis of data suggests that adverse drug reactions accounted for nearly 100000 human deaths and more than two million hospitalizations in 1994 (9). Recognizing the potential public benefit as well as their own common interest in SNPs, members of The SNP Consortium (TSC; http://snp.cshl.org/; see A. Holden, this Supplement) began in 1998 "...to develop up to 300000 SNPs distributed evenly throughout the human genome, and to make the information related to these SNPs available to the public without intellectual property restrictions" (5). This organization of eleven major pharmaceutical and technology companies, four major genomics centers and the Wellcome Trust eventually mapped close to a million SNPs by the end of 2001. But much as the human genome project to sequence the entire human genome was just a beginning step toward understanding our own complicated life processes, so too was the effort to produce a high-density SNP map the beginning of our understanding of genetic variation at the primary sequence level.

SNPs are regarded as one of the keys to predictive pharmacogenomic medicine. This proactive predictive ability will aid physicians in making diagnoses and knowing in advance (i) which of their patients are susceptible to specific diseases, (ii) which medical treatments are likely to be of greatest benefit, and (iii) which treatments are ones to avoid (11). For a SNP to be of clinical or economic value, future researchers will need to know what are the causative SNPs and which ones correlate with diagnoses (1). This knowledge will require information about the many different SNP sites on the three billion base pair human genome to become known and available. In the following sections, this article briefly discusses efforts to fulfill these requirements from a market point of view and presents the commercial technologies of companies offering products for SNP genotyping.

\section{STEPS IN SNP RESEARCH}

Research interest in any particular SNP begins with its discovery, which is usually accomplished by comparing primary sequence data between any two or more genetically distinct individuals. The SNP Consortium's Web site lists the sequencing methods used to develop that association's SNP map. Errors in the sequencing process can lead to the incorporation of false positives in a SNP database, so the second step of SNP research is confirmation: the determination of whether there are in fact two or more alleles at an identified SNP site, with variation arising at a frequency to be of practical use (usually more than a couple percent). Next, researchers must determine whether the SNP is potentially useful. This is usually done by linkage analysis. If a SNP is found to have potential utility, the final step is application, where the SNP is used to stratify patients for a clinical trial, to diagnose disease or disease susceptibility, or to evaluate a patient's genetic make-up to guide the selection of appropriate drug therapy.

Within each of these stages is an abundance of opportunities for the creative application of technology to SNP research. Manufacturers of genetic analysis systems have taken up the challenge, combining many different methods to produce an exciting array of instrumentation, software, and reagent systems to address each stage. At present a lot of effort is focused on SNP confirmation and validation, since pharmaceutical companies 
are willing to pay for validated genetic targets in key therapeutic areas. In addition to forming SNP project collaborations with pharmaceutical companies, some SNP-analysis system manufacturers, such as Sequenom (http://www.sequenom.com) and Orchid BioSciences (http://www.orchid.com) are applying their in-house SNP analysis capacities to the development of valueadded databases consisting of confirmed SNPs and preliminary SNP validation based on ethnicity, allele frequency, and gene structure. These databases can both accelerate and increase the manufacturers' return on investment in product development, and serve as a marketing vehicle to showcase the capabilities of products that either are, or soon will be, for sale.

The final step, application, is characterized by analysis system requirements quite different from those of the confirmation and validation stages. SNP confirmation and validation projects can require that tens of thousands of samples from individuals be screened over many SNPs, producing a project throughput requirement of millions of individual SNP scores. By comparison, a SNP diagnostic application may require scoring just a few SNPs in a single individual to determine whether a certain treatment will be safe to use in a particular individual or if an individual is at risk for developing an adverse response.

A high level of automation is required to achieve the sort of high-throughput levels required for confirmation and validation. These automated processes are typically attended closely by skilled lab personnel that know how to keep the equipment running optimally and move sample collections and data from one step to the next. To be viable in the clinic, though, a SNP analysis system has to be simple to operate. Therefore if the major market participants in the confirmation and validation stages of SNP studies choose also to participate in the diagnostic stage, they will need to figure out how to modify the operation of their systems to achieve a level of technical simplicity comparable with that of other clinical diagnostic instrumentation.

\section{ASSEMBLING THE TECHNOLOGY}

Automation in SNP analysis has been greatly facilitated through the use of liquid handling robots, plate movers, various kinds of "hotels," incubators, and rails. Commercial SNP scoring often begins with an amplification reaction designed to immediately or eventually disclose which nucleotide occupies a given SNP site in a given sample. In some assays, the amplification reaction is directly coupled to the SNP analysis; in other types of assays a secondary assay is needed to determine the allele. Several instrumentation-based detection technologies can be used to read the result. Software for directing the sample and data flow, running the instrumentation, and collecting and analyzing data completes a typical system.

Many SNP assays used to determine a SNP allele fall into one of two major classes: those that use an abbreviated form of DNA sequencing technology, and those that use basic hybridization affinity between perfectly and imperfectly complementary DNA strands as a basis for determining the identity of the SNP site nucleotide. Processes based on DNA sequencing technology exploit the specificity of a non-proofreading DNA polymerase that enables that enzyme to identify, select, and incorporate the nucleotide complementary to the DNA strand op- posite a primed DNA template. These sequencing-based methods themselves fall into one of two categories: those that place a primer immediately upstream of the allele in question and incorporate a single nucleotide at the SNP site, and those that simply place a primer near the SNP site, and allow the sequencing reaction to proceed to, or through, the SNP site.

For the single base extension reactions, methods for determining which nucleotide has been incorporated at the SNP site include colorimetric and fluorescence-based detection, or mass determination of the extended primer. A commercial application of SNP analysis by multiple-base primer extension, Pyrosequencing $^{\mathrm{TM}}$, couples a light-emitting reaction to nucleotide incorporation.

Hybridization-based methods also fall into two main categories: those that use differential hybridization stringency alone to determine which nucleotide occupies the SNP site, and those that combine hybridization with an enzyme that recognizes specific hybridized structures. Detection schemes are fluorescence-based.

\section{PRODUCING PRODUCTS}

Orchid BioScience's SNP-IT single-base primer extension technology is one that makes use of a DNA polymerase enzyme to identify which allele is present at a particular site (this Supplement). Sometimes referred to as single-base extension, or "mini-sequencing," SNP-IT based methods begin with amplified target sequence and the placement of a primer immediately $5^{\prime}$ to the SNP site. Under sequencing reaction conditions with chain-terminating nucleotides, DNA polymerase adds a single, labeled base. The identity of the base can be determined using a variety of methods, including colorimetry, fluorescence, and mass spectrometry. The identity of this base reveals the allelic variant of the SNP.

Michael Boyce-Jacino, PhD, Orchid's Chief Technology Officer and Vice President of R\&D acknowledges that large-scale SNP confirmation, linkage disequilibrium, and genome-wide haplotyping studies are key applications for Orchid's SNP-IT technology, but says that rather than position the technology as optimal for one or more specific SNP applications, the company's goal is to establish the technology on as many platforms as possible and roll it out to the market through their platform partners' customer bases. Orchid's SNP-IT technology is behind brand-name SNP analysis kits such as Amersham Biosciences' SNuPe ${ }^{\mathrm{TM}}$ Genotyping Kit (http://www.amershambiosciences. com) for analysis on the MegaBACE capillary DNA analysis platform, and Applied Biosystems' SNaPshot ${ }^{\mathrm{TM}}$ Multiplex Kit (http://www.appliedbiosystems.com) for analysis on ABI PRISM $^{\circledR}$ capillary electrophoresis instruments. SNP-IT technology also works with Orchid's own SNPstream ${ }^{\mathrm{TM}}$ instrumentation, fluorescence detection platforms from Affymetrix (http:// www.affymetrix.com) and Luminex (http://www.luminexcorp. com), and fluorescence polarization detection systems such as Molecular Devices' Analyst ${ }^{\mathrm{TM}}$ multi-mode detection system (http://www.moleculardevices.com). The greatest market advantage of SNP-IT technology may be that the biochemistry is adaptable to a variety of reporter chemistries and readout devices. Looking forward, Dr. Boyce-Jacino believes that the biggest payoff in the SNP market is going to be in the clinical 
application stage, which will include point-of-service diagnostics. Therefore, Orchid's goal, he says, is to "make sure the technology is so ingrained that it's there at the end."

Pyrosequencing, a DNA sequencing technology commercialized by Pyrosequencing $\mathrm{AB}$ of Sweden (http://www.pyrosequencing.com), also uses DNA polymerase in a sequencing reaction to make the SNP call, but the reaction proceeds beyond one nucleotide to provide Pyrosequencing users with short-run sequencing data around their SNPs (10). In Pyrosequencing, a sequencing primer positioned upstream of the SNP site is incubated with amplified target sequence, DNA polymerase, and an enzyme cocktail that releases light by measuring the amount of pyrophosphate released when a nucleotide is incorporated into the primed DNA strand. Pyrosequencing AB's PSQ ${ }^{\mathrm{TM}}$ sequence analysis systems add the four-deoxynucleotide triphosphates one at a time and measures the light signals that ensue when nucleotides are incorporated. The PSQ system records the order of incorporated nucleotides in the extending primer by correlating light emissions with the timing of deoxynucleotide triphosphate addition. The PSQ systems can read out to 40 nucleotides without labeling, fragment separation, or off-line detection instrumentation.

One of the key strengths of Pyrosequencing technology, according to Theresa McNeely, Senior Director of PR and IR at Pyrosequencing $\mathrm{AB}$, is that it scores SNPs in the context of surrounding sequence, rather than providing a single nucleotide data point, providing assurance that the SNP genotype reported is, in fact, the nucleotide at the site of interest on the DNA template. Kellie Watson, PhD, Director of Business Development at Pyrosequencing $\mathrm{AB}$, points out that Pyrosequencing is also appropriate for scoring tri- and tetra-allelic SNPs since the technique doesn't require any assumptions about nucleotide identities, and with the ability to sequence short runs, one can "get haplotype information all in one read." Dr. Watson also mentions primer flexibility as a key advantage of Pyrosequencing. "A potential challenge for customers is a SNP in a difficult location," says Dr. Watson. "With Pyrosequencing, you can place your primer wherever it's optimal relative to the SNP, up to 30 nucleotides away. We're always able to design primers for assays."

Sequenom is specifically targeting the market for highthroughput SNP scoring applications with their MassARRAY ${ }^{\mathrm{TM}}$ system, starting with SNP confirmation studies designed to remove false positives from SNP databases and determine allele frequencies. "You have to know which SNPs are real, are what are their actual frequencies, otherwise you're wasting time, effort and money," says Tristan Orpin, Senior Vice President of Sales and Marketing at Sequenom. Additional high-throughput projects in the MassARRAY portfolio include linkage disequilibrium studies, large-scale disease association studies and population stratification studies based on baseline factors such as ethnicity, sex, and age. "If a potential customer is looking only at doing small-scale studies, we let them know they should look somewhere else," says Mr. Orpin.

The MassARRAY system works with Sequenom's MassEXTEND $^{\mathrm{TM}}$ chemistry, which uses homogenous extension primers, DNA polymerase, and dideoxynucleotide termination to generate extension products for mass spectrometry analysis (this Supplement). Sequenom's SpectroTYPER-RT software calls SNP genotypes based on the mass spectrometry data. According to Mr. Orpin, the technology addresses both the accuracy and cost con- cerns of scientists doing high-throughput studies: separation and analysis of extension products by mass spectrometry produces high accuracy data, and consumable costs per reaction are maintained at economical levels since there are no beads, specialized primers, or labels. Sequenom also seeks to establish the company's market position on the basis of the scientific support available to customers. "Our customers include the top centers for genetic analysis," says Mr. Orpin. "We can't have a bad reference site."

Technology from Third Wave Technologies (http://www. twt.com) offers an example of a combined hybridization and enzyme recognition approach to SNP scoring (4). Called the "Invader $^{\circledR}$ Operating System," Third Wave's system works by hybridizing a short oligonucleotide probe to target DNA. A second "invader" oligonucleotide partially displaces the 5 ' end of the primary probe to produce a flap, which is cleaved off and used to induce a conformational change in Third Wave's fluorescence resonance energy transfer molecule ("FRET cassette"). Once associated with a flap from the cleaved primary probe, the FRET cassette is cleaved, unquenching its fluorophore. In the presence of a complementary primary probe, the target DNA can give rise to thousands of unquenching reactions over time, enabling fluorescent signal accumulation and measurement. Third Wave's "biplex" assay format detects multiple allelic SNP variations and enables complete SNP genotyping in a single reaction.

Since the fluorescent signal accumulates over time, Third Wave's Invader Operating System can call genotypes without the benefit of target DNA amplification. "It's the only technology that is PCR independent," says Lance Fors, PhD, Chief Executive Officer at Third Wave. According to Dr. Fors, the ability to work directly on genomic DNA, and the overall simplicity and robustness of the Invader Operation System chemistry are key advantages enabling Third Wave to carry their technology all the way to clinical diagnostics laboratories. "As you get closer to the point of care, the advantage goes to the technology with fewer steps," says Dr. Fors. "Our chemistry is very simple-just add genomic DNA to the well."

Dr. Fors says Third Wave has produced tens of thousands of individual SNP genotyping assays for research based on the Invader Operating System and has developed ten genotyping kits for use in clinical DNA diagnostics laboratories, giving the company a leading position for genotyping products in the clinical market.

Another SNP technology making use of enzymatic specificity is the oligonucleotide ligation assay (OLA) (7). In OLA, DNA ligase is used to covalently join two oligonucleotides aligned adjacent to one another along a complementary strand of amplified target DNA. The first oligonucleotide, a "capture" probe, contains a 25-mer ZipCode sequence and an allele-complementary sequence representing one of the genotypes under analysis. The second oligonucleotide is a fluorescence-labeled "reporter" probe. If the allele complementary sequence is complementary to the target DNA at the SNP position, DNA ligase will join it to the fluorescent reporter probe. DNA ligase requires perfect end-to-end alignment and will not join the capture probe and the reporter probe if the capture probe is not complementary to the target DNA at the SNP query position. The ligation product is hybridized to the complementary ZipCode sequence "cZipCode" coupled via a carbon chain standoff to a fluorescent microsphere. Ligation assay results are read in a flow cytometer.

Similar approaches substituting allele-specific primer exten- 
sion (ASPE), and single base chain extension (SBCE) in place of OLA have been published $(2,16)$. According to Michael Weiner, $\mathrm{PhD}$, Vice President of Molecular Sciences at 454 Corporation, a derivation of a microsphere-based system developed by GlaxoWellcome Research and Development, using SBCE, has been commercially developed for the research market by collaboration between Orchid BioSciences and Luminex Corporation. Researchers can assemble OLA and ASPE assays from published descriptions (13).

Illumina (http://www.illumina.com) has described the application of an OLA assay for SNP scoring to their fiber optic BeadArray ${ }^{\mathrm{TM}}$ platform $(6,15$; this Supplement). Illumina's technology uses microspheres with attached "address" sequences to immobilize fluorescent ligation products for analysis, but rather than using a flow cytometer, Illumina's technology uses microspheres bearing a number of different address sequences to selfassemble in the cupped ends of individual fibers at the end of a fiber optic bundle. The fiber optic format specifies a dense microsphere arrangement while enabling distinct fluorescence interrogation of each microsphere using confocal optics. The stable association of one bead per fiber permits Illumina to use a two-step hybridization assay with fluorescence-labeled oligonucleotides complementary to the address sequences to identify address sequences attached to each bead held at the end of the fiber optic bundle.

Illumina has described using fiber optic bundles composed of 50000 fibers, each representing a potential assay sensor. Illumina multiplies the platform architecture by arranging the fiber optic bundles themselves in the industry-standard 96 element array pattern to yield an "array of arrays" with millions of sensors.

"We're not interested in low sample throughput," states Bill Craumer, Director of Marketing Communications at Illumina. The company sees a market requiring "billions of tests" in the near future, and places themselves in the market space for applications such as linkage disequilibrium studies requiring hundreds of thousands of SNP scores on hundreds of thousands of samples. The company is preparing to commercialize their SNP genotyping platform through an instrumentation development collaboration with Applera's Applied Biosystems Group.

\section{SCALING UP}

At present, the main focus in SNP analysis system product development is on increasing throughput while maintaining both high accuracy and driving down the cost per genotype. Developers of SNP analysis systems have invented a number of remarkable ways to get more information out of their systems in less time by multiplexing sample throughput, automating sample preparation, or both.

Since it relies on oligonucleotide reaction primers, Orchid's SNP-IT technology offers a number of multiplexing opportunities based on primer size or label, depending on the readout platform with which the technology is paired. According to Dr. Boyce-Jacino, the question with respect to multiplexing is, "how to get dozens of assays to work together" in a single reaction. Orchid is conducting an in-house informatics project focused on primer design methods that will increase the throughput on their partner's platforms by enabling dozens of SNPs to be assayed together.
Amersham Biosciences' SNuPe assay uses Orchid's singlebase extension technology with four different dye terminators to determine the identity of the added nucleotide. The SNuPe assay is read in Amersham's MegaBACE parallel capillary electrophoresis platform, which can load and read up to 384 capillary separations at once. Amersham multiplexes this throughput by injecting each capillary multiple times.

Pyrosequencing's PSQ platform is designed to read 96 Pyrosequencing reactions at once. In addition, Pyrosequencing's company literature describes a multiplexing scheme that enables three SNP sites to be read in a single reaction (14). The company reports success with additional levels of multiplexing. The multiplexing method uses a separate sequencing primer for each polymorphism, and the order of nucleotide addition is arranged to simplify the interpretation of Pyrograms produced during Pyrosequencing. The company is also working closely with TECAN to automate sample preparation.

System manufacturers match their multiplexing strategies to the capabilities of their readout devices. The fine mass discrimination capability of mass spectrometry enables Sequenom's MassARRAY system to sort multiplexed sequencing reactions primed with differently sized oligonucleotides. Of the makers of SNP analysis systems, Sequenom has made the most visible investment in sample automation, partnering with German industrial automation powerhouse Grohmann Engineering (http:// www.grohmann-engineering.de) to produce the MassARRAY 200K system, announced in March of 2002.

Third Wave Technologies' Invader assay derives its speed advantage by working directly on genomic DNA. Unlike other methods, Invader assay throughput is not limited by PCR setup and cycling time. The company works with Beckman Coulter (http://www.beckmancoulter.com) and TECAN (http: //www.tecan.com) on high-throughput automation development. The company's clinical test development and commercialization partner, BML (http://www.bml.co.jp), uses in-house developed automation systems to support their high-throughput Invader assay applications.

SNP genotyping using OLA and flow cytometric detection makes use of fluorescent microspheres as a substrate for the cZipCode sequences that capture OLA ligation products. The fluorescent microspheres are available in different ratios of red and orange fluorescence, enabling discrimination of up to 100 different microsphere types during flow cytometric analysis. By attaching different cZipCode sequences to different microsphere types, researchers can multiplex their OLA-based SNP genotyping assays $(7,13)$.

The microspheres used by Illumina with their fiber-optic BeadArray platform have no intrinsic fluorescence, according to David Barker, PhD, Vice President and Chief Scientific Officer at Illumina. Microsphere identities are decoded on the basis of complementary sequence hybridizations to their attached "address" sequences. In practice, the company aims for 25 -fold redundancy in microsphere array self-assembly, yielding 2000 unique address sequences at the end of each 50000 -element fiber optic. Illumina states that by multiplexing the OLA assay and using a different fluorescent reporter for each SNP allele, they were genotyping 96 SNPs simultaneously at the end of one fiber optic array. Dr. Barker reports that the company has continued to achieve progressive increases in multiplexing throughput. 
Table 1. Commercially Available SNP Products and Services

\begin{tabular}{|c|c|}
\hline Company Name & Products and Services \\
\hline Affymetrix & $\begin{array}{l}\text { Instrumentation: GeneChip }{ }^{\circledR} \text { Fluidics Station, GeneArray }{ }^{\circledR} \text { Scanner } \\
\text { Software: GeneChip }{ }^{\circledR} \text { Analysis Software, Microarrray Suite Software, HuSNP }{ }^{\text {TM Mapping Assay }} \\
\text { Consumables: GeneChip HuSNP Probe Array, GeneChip HuSNP Reagent Kit, GenFlex }{ }^{\mathrm{TM}} \\
\quad \text { Tag Array }\end{array}$ \\
\hline Alpha Innotech & Instrumentation: AlphaArray ${ }^{\mathrm{TM}}$ High Throughput Microarray Reader \\
\hline Amersham Biosciences & $\begin{array}{l}\text { Instrumentation: MegaBACE }{ }^{\mathrm{TM}} \text { DNA Analysis System } \\
\text { Consumables: MegaBACE SNuPe }{ }^{\mathrm{TM}} \text { Genotyping Kit }\end{array}$ \\
\hline Applied Biosystems & $\begin{array}{l}\text { Instrumentation: ABI PRISM }{ }^{\circledR} \text { capillary electrophoresis instruments, ABI PRISM } 7000 \text { Sequence } \\
\quad \text { Detection System } \\
\text { Software: GeneMapper }{ }^{\mathrm{TM}} \text { Software, Genotyper }{ }^{\circledR} \text { Software, SeqScape }{ }^{\mathrm{TM}} \text { Software } \\
\text { Consumables: SNaPshot }{ }^{\mathrm{TM}} \text { Multiplex Kit } \\
\text { Services: Assays-by-DesignSM Service }\end{array}$ \\
\hline Gentris & Services: Research Genotyping, Clinical Genotyping, HIV Genotyping \\
\hline Intergen & Consumables: Amplifluor ${ }^{\mathrm{TM}}$ SNP Genotyping System \\
\hline Illumina & Services: SNP Genotyping Services \\
\hline Marligen Biosciences & Consumables: Signet ${ }^{\mathrm{TM}}$ SNP Array Kits \\
\hline Molecular Devices & $\begin{array}{l}\text { Instrumentation: Analyst }{ }^{\mathrm{TM}} \text { multi-mode detection system } \\
\text { Software: AlleleCaller }{ }^{\mathrm{TM}} \text { software }\end{array}$ \\
\hline Nanogen & $\begin{array}{l}\text { Instrumentation: NanoChip Molecular Biology Workstation } \\
\text { Consumables: clinical genotyping assay }\end{array}$ \\
\hline Orchid BioSciences & $\begin{array}{l}\text { Instrumentation: SNPScope }{ }^{\mathrm{TM}} \text { Array Imager, SNPStream }{ }^{\circledR} \text { Instruments } \\
\text { Software: SNPstream UHT Software Suite, GetGenos }{ }^{\mathrm{TM}}, \mathrm{QCreview}^{\mathrm{TM}} \text {, ValidGenes } \\
\text { software } \\
\text { Consumables: SNPware }{ }^{\mathrm{TM}} \text { SNP Scoring Kits, SNPcode } \\
\text { Services: MegaSNPatron facility genotyping services, custom assay development, diagnostic } \\
\text { and forensic services }\end{array}$ \\
\hline Pyrosequencing $\mathrm{AB}$ & $\begin{array}{l}\text { Instrumentation: PSQ }{ }^{\mathrm{TM}} \text { Systems } \\
\text { Software: SNP Software AQ } \\
\text { Consumables: PSQ } 96 \text { SNP Reagent Kits }\end{array}$ \\
\hline Sequenom & $\begin{array}{l}\text { Instrumentation: MassARRAYTM System } \\
\text { Software: SpectroDESIGNER } \\
\quad \text { SpectroTYPER Assay Design Software, SpectroCHECK }{ }^{\mathrm{TM}} \text { RT Software SpectroTYPER Database Software } \\
\text { Consumables: SpectroCHIP System, } \\
\text { Services: SNP genotyping collaborations } \\
\text { Data: RealSNP.com }{ }^{\mathrm{TM}} \text { SNP assays }\end{array}$ \\
\hline Third Wave Technologies & $\begin{array}{l}\text { Consumables: Invader } \mathrm{TM} \text { genotyping kits for life science research, Invader clinical reagents } \\
\text { Services: Custom Invader assays }\end{array}$ \\
\hline
\end{tabular}

\section{SCALING DOWN}

At present, the money in the noisy market for SNP analysis systems is on developing and ramping up the throughput of highly automated, highly multiplexing systems that can deliver the millions of accurate SNP scores pharmaceutical researchers need to conclusively connect specific genotypes and haplotypes to clinical phenotypes within an economical time frame. As pharmacogenomics-based medicine becomes a more conventional element of health care, the demand for massively high- throughput systems will not likely diminish. But easy-to-use, low-throughput systems for analyzing the SNPs of individuals, at or near the point of care, will also become a necessary component of health care delivery. The market specifications for these systems are quite different that the ones that are now being developed for high-throughput SNP scoring applications. For the clinical diagnostics laboratory, a system may need to score only a few SNPs and handle just one sample at a time.

Similar changes will occur within pharmaceutical research itself as projects progress from SNP discovery to validation of 
SNP markers. That progression will be marked by a significant reduction in the number of SNP loci interrogated and a dramatic increase in the number of samples assayed. Sherry Challberg, $\mathrm{PhD}, \mathrm{CEO}$ of Marligen Biosciences Inc., says that Marligen's Signet ${ }^{\mathrm{TM}}$ SNP Array products utilize a suspension array technology that can be multiplexed up to 100-fold and are compatible with automation. Marligen sells Signet SNP Array kits for human mitochondrial DNA and Y chromosome SNPs, and offers custom SNP Array kits and services.

Some of the current makers of SNP analysis systems clearly aspire to participate in the upcoming clinical stage of the SNP market. Third Wave Technologies, for instance, has licensed rights to develop clinical applications based on 3300 genetic markers within approximately 200 genes that regulate drug metabolism, and has already released a number of kits for use in clinical diagnostics laboratories. Pyrosequencing has developed a SNP-based intellectual property portfolio around cardiovascular disease diagnostics. Unsurprisingly, it appears as though the manufacturers with the most visible investments in developing SNP applications for the clinic are the ones offering the easiest-to-use assay formats and instrumentation. But DNA-based assays are still fairly uncommon in clinical diagnostics laboratories.

Gentris Corporation (http://www.gentris.com) is a new company formed for the purpose of commercializing clinical pharmacogenomics products, including genotyping kits and validated reference controls for SNP genotyping of drug metabolizing enzymes. Presently, Gentris offers general purpose reagents for SNP diagnostics in clinical reference laboratories. According to Michael Murphy, President and Chief Executive Officer of Gentris, a "different mindset is required" when it comes to developing products for the clinic. A molecular biology research laboratory may have, or make routine use of, a variety of instrumentation for tasks such as oligonucleotide synthesis, DNA amplification, electrophoretic separation and mass spectrometry analysis. Clinical diagnostics laboratories are more typically equipped with instrumentation that accepts a closed clinical specimen, a tube of blood for instance, at one end, and delivers a printed result at the other, with no human intervention in between. Mr. Murphy believes that Nanogen (http://www.nanogen.com) is on the right track with their NanoChip ${ }^{\mathrm{TM}}$ microelectronic array systems, which can be programmed to set up and carry out multiple-step biological assays in a closed, automated environment. With the market settling out according to research and clinical applications, it's unclear which manufacturers command SNP analysis technologies that will scale and adapt to succeed at both. At present, the market landscape is incompletely defined and offers a variety of viable positions for makers of SNP analysis systems.

\section{REFERENCES}

1.Branca, M.A. 2002. Linking variation to disease and response genes. Drug Disc. Dev. 5:47-50

2.Chen, J., M. Iannone, M. Li, J. Taylor, P. Rivers, A. Nelson, K. SlentzKesler, A. Roses, et al. 2000. A microsphere-based assay for multiplexed single nucleotide polymorphism analysis using single base chain extension. Genome Res. 10:549-557.

3.Cooper, D.N., B.A. Smith, H.J. Cooke, S. Niemann, and J. Schmidtke. 1985. An estimate of unique DNA sequence heterozygosity in the human genome. Hum. Genet. 69:201-205.
4.Hall, J.G., P.S. Eis, S.M. Law, L.P. Reynaldo, J.R. Prudent, D.J. Marshall, H.T. Allawi, A.L. Mast, et al. 2000. Sensitive detection of DNA polymorphisms by the serial invasive signal amplification reaction. Proc. Natl. Acad. Sci. USA 97:8272-8277.

5.http://snp.cshl.org.

6.http://www.illumina.com/Randomly_Assembled_Arrays_Genotyping.pdf.

7.Iannone, M.A., J.D. Taylor, J. Chen, M. Li, P. Rivers, K. Slentz-Kesler, and M. Weiner. 2000. Multiplexed single nucleotide polymorphism genotyping by oligonucleotide ligation and flow cytometry. Cytometry 39:131-140.

8.Kruglyak, L. and D.A. Nickerson. 2001. Variation is the spice of life. Nat. Genet. 27:234-2366.

9.Lazarou, J., B.H. Pomeranz, and P.N. Corey. 1998. Incidence of adverse drug reactions in hospitalized patients. A metaanalysis of prospective studies. JAMA 279:1200-1205.

10.Ronaghi, M., Uhlen, M., and P. Nyrén. 1998. A sequencing method based on real-time pyrophosphate. Science 281:363-365.

11.Roses, A.D. 2000. Pharmacogenetics and the practice of medicine. Nature 405:857-865.

12.Sachidanandam, R., D. Weissman, S.C. Schmidt, J.M. Kakol, L.D. Stein, G. Marth, S. Sherry, J.C. Mulliken, et al. 2001. A map of human genome sequence variation containing 1.42 million single nucleotide polymorphisms. Nature 409:928-33.

13.Taylor, J.D., D. Briley, Q. Nguyen, K. Long, M. Iannone, M. Li, F. Ye, A. Afshari, et al. 2001. Flow cytometric platform for high-throughput single nucleotide polymorphism analysis. BioTechniques 30:661-669.

14.www.pyrosequencing.com Technical note 105 .

15.www.tigr.org.

16.Ye, F., M-S Li, J.D. Taylor, Q. Nguyen, H. Colton, W. Casey, M. Wagner, M. Weiner, et al. 2001. Fluorescent microsphere-based readout technology for multiplexed human single nucleotide polymorphism analysis and bacterial identification. Hum. Mutat. 17:305-316.

\section{Address correspondence to:}

Matthew Wygant

Matthew Wygant Biotech Writing

416 Los Gatos Boulevard

Los Gatos, CA 95032

e-mail: matthew@wygant.net 